\title{
CONTINUA AS POSITIVE WHITNEY LEVELS
}

\author{
WLODZIMIERZ J. CHARATONIK
}

(Communicated by James E. West)

\begin{abstract}
It is shown that every continuum is a positive Whitney level of some continuum.
\end{abstract}

Krasinkiewicz and Nadler have asked (independently) if every continuum is a positive Whitney level of some continuum. The question has never been published, but it is known for people working in continuum theory. Here we present a very simple proof of it, which is in fact a compilation of known results.

For a given continuum $X$ we denote by $C(X)$ the hyperspace of all subcontinua of $X$ with the Hausdorff metric. A Whitney map is a map $\omega: C(X) \rightarrow$ $[0, \infty)$ that satisfies the following two conditions:

(1) $\omega(\{x\})=0$ for all $x \in X$, and

(2) if $A, B \in C(X)$ satisfy $A \subseteq B$ and $A \neq B$ then $\omega(A)<\omega(B)$.

We will also use the notion of an atomic continuum. A subcontinuum $A$ of a continuum $X$ is called atomic if for any subcontinuum $B$ of $X$ we have $A \subseteq B$ or $B \subseteq A$. In newer papers atomic continua are also called terminal.

Theorem. Let $X$ be any continuum. Then there exist a continuum $M, a$ Whitney map $\omega: C(M) \rightarrow[0, \infty)$, and a number $t \in(0, \omega(M))$ such that $X$ is homeomorphic to $\omega^{-1}(t)$.

Proof. By [1, Theorem, p. 507] there exist a continuum $M$ and a monotone open map $f: M \rightarrow X$ such that all point inverses $f^{-1}(x)$ for $x \in X$ are nondegenerate atomic subcontinua of $M$. Let

$$
\mathscr{M}=\{\{m\}: m \in M\} \cup\left\{f^{-1}(x): x \in X\right\} \cup\{M\} .
$$

Thus $\mathscr{M}$ is a compact subset of $C(M)$. Define $w: \mathscr{M} \rightarrow[0, \infty)$ by $w(\{m\})=$ 0 , for $m \in M, w\left(f^{-1}(x)\right)=1$, and $w(M)=2$. Then $w$ is continuous, and by [3, Corollary 3.4, p. 468] it can be extended to a Whitney map $\omega: C(M) \rightarrow[0, \infty)$. Because all point inverses $f^{-1}(x)$ for $x \in X$ are atomic continua, we have $\omega^{-1}(1)=\left\{f^{-1}(x): x \in X\right\}$ and therefore $\omega^{-1}(1)$ is homeomorphic to $X$. The proof is complete.

Received by the editors March 27, 1992.

1991 Mathematics Subject Classification. Primary 54B20.

Key words and phrases. Atomic decomposition, continuum, hyperspace, Whitney level, Whitney map.

(C) 1993 American Mathematical Society $0002-9939 / 93 \$ 1.00+\$ .25$ per page 


\section{ACKNOWLEDGMENT}

The author would like to thank Pawel Krupski for help in finding the appropriate reference.

\section{REFERENCES}

1. R. D. Anderson, Atomic decompositions of continua, Duke Math. J. 23 (1956), 507-514.

2. S. B. Nadler, Jr., Hyperspaces of sets, Dekker, New York, 1978.

3. L. E. Ward, Jr., Extending Whitney maps, Pacific J. Math. 93 (1981), 465-469.

Institute of Mathematics, University of Wroclaw, Pl. Grunwaldzki 2/4, 50-384 Wroclaw, Poland

E-mail address: wjcharat@plwruw11.bitnet 